\title{
Réanalyses avec et sans convergence : l'imbrication des facteurs sociaux, communicationnels et structurels dans le contexte plurilingue ivoirien
}

Reanalyses with and without convergence: the interweaving of social, communicational and structural factors in the plurilingual Ivorian context

\section{Akissi Béatrice Boutin}

\section{OpenEdition}

Journals

Édition électronique

URL : https://journals.openedition.org/tipa/4445

DOI : $10.4000 /$ tipa. 4445

ISSN : 2264-7082

Éditeur

Laboratoire Parole et Langage

Référence électronique

Akissi Béatrice Boutin, « Réanalyses avec et sans convergence : l'imbrication des facteurs sociaux, communicationnels et structurels dans le contexte plurilingue ivoirien », TIPA. Travaux interdisciplinaires sur la parole et le langage [En ligne], 37 | 2021, mis en ligne le 31 août 2021, consulté le 20 octobre 2021. URL : http://journals.openedition.org/tipa/4445; DOI : https://doi.org/10.4000/tipa. 4445

Ce document a été généré automatiquement le 20 octobre 2021.

\section{(†) $९$}

La revue TIPA. Travaux interdisciplinaires sur la parole et le langage est mise à disposition selon les termes de la licence Creative Commons Attribution - Pas d'Utilisation Commerciale - Pas de Modification 4.0 International. 


\title{
Réanalyses avec et sans
}

convergence : l'imbrication des facteurs sociaux,

communicationnels et structurels dans le contexte plurilingue ivoirien

\author{
Reanalyses with and without convergence: the interweaving of social, \\ communicational and structural factors in the plurilingual Ivorian context
}

Akissi Béatrice Boutin

\section{Introduction 1}

1 L'avantage des situations plurilingues et pluriculturelles dans l'Afrique d'aujourd'hui est qu'elles sont observables et vécues en direct et non à reconstituer à partir de recherches dans le passé. De ce fait, l'apport de la linguistique africaine aux études sur le changement linguistique est immense et elle permet souvent de reconsidérer les catégories et conceptualisations des langues et des pratiques issues du contact (Nunez \& Léglise 2016; Ebongue \& Hurst, 2017; Boutin \& Kouadio N'Guessan 2015). En particulier, des études fines qui appréhendent l'écologie des activités et des espaces où les langues sont observées mettent en lumière une multiplicité de phénomènes dus à l'omniprésence de la variation intra et inter langues, en ville et ailleurs.

Dans ce texte nous nous penchons sur les influences entre les langues sur une aire plurilingue, dans le but de questionner la notion de convergence. Cette notion présente l'intérêt de tenir compte d'emblée du fait que plusieurs facteurs ensemble peuvent créer une situation propice au changement du fait même de leur réunion, celui-ci étant 
toujours multi-causal (Mufwene 2008; Ludwig, Pagel \& Mühlhäusler 2018). En Côte d'Ivoire, de nombreux phénomènes du français, du dioula et du baoulé en contact manifestent deux niveaux de convergences : d'abord une convergence entre plusieurs facteurs d'ordre sociolinguistique et communicationnel qui favorisent l'apparition d'une innovation dans une langue (Kriegel 2018; Mufwene 2005), ensuite entre les langues, lorsqu'un schéma de construction porteur de sens dans une ou plusieurs de ces langues a servi de modèle pour une réanalyse des formes dans une ou plusieurs autres langues. La notion de réanalyse que nous utilisons ici est proche de celle de refonctionnalisation et se conçoit aisément à la suite de processus d'acquisition non guidés. La réanalyse est le résultat de stratégies de compréhension, une réinterprétation du lexique et du rôle de morphèmes ou de constructions (Cf. Kriegel 2003 ; Detges 2003) qui aboutit à une innovation incluant une recatégorisation.

3 Notre point de vue est essentiellement grammatical, mais avec la prudence qui convient devant des variétés (ou des langues) changeant très vite, sur des aires postcoloniales où la langue européenne a totalement modifié les rapports entre les langues présentes antérieurement. En outre, les facteurs structurels, individuels et sociaux se mêlent non seulement dans les interférences dues aux contacts, mais bien avant dans les pratiques langagières (Gumperz 1964 ; Gumperz \& Wilson 1971 ; Winford 2003 ; Léglise 2018). Les faits considérés sont donc à voir comme des phénomènes qui manifestent des réanalyses dans les pratiques langagières et tendent à s'élaborer en système.

4 Après avoir examiné la convergence des facteurs pour la Côte d'Ivoire (1), nous étudierons plus en détail deux cas de réanalyses assez différents dans le but d'explorer une possible convergence des langues: la possibilité pour des syntagmes prépositionnels d'avoir des fonctions de syntagmes nominaux (2), avec le cas particulier de pour (3), et la fonctionnalisation d'une forme verbale comme conditionnel périphrastique en français (4). Nous nous demandons si cette notion est pertinente pour expliquer des réanalyses observées dans le français ou les langues en contact.

\section{Des facteurs sociaux et communicationnels qui favorisent la convergence des langues}

5 Les facteurs susceptibles de converger dans les sociétés plurilingues, et d'être à l'origine de changements dans les langues sont de plusieurs ordres: sociaux, individuels, structurels, ou ils font partie de tendances plus larges qui affectent toute langue. Nous verrons ici comment ces facteurs peuvent s'exercer dans une aire plurilingue comme la Côte d'Ivoire. Toutefois, les sources structurelles de convergence seront illustrées dans les sections suivantes (2 et 3 ).

6 Des facteurs sociaux sont, dans le cadre général de la politique linguistique du pays, des éléments plus précis comme l'influence des langues de plus grande diffusion, la tolérance à l'intercompréhension approximative, la popularité d'un argot métissé, et les pratiques plus ou moins consensuelles d'alternances de langues.

7 Un premier facteur est la vitalité des langues politiquement minorées alors qu'une autre langue est imposée, en l'occurrence le français. La politique linguistique ivoirienne, qui découle de choix politiques et économiques plus généraux liés au 
contexte postcolonial, se résume, depuis la colonisation, à l'omniprésence du français et l'ignorance de la soixantaine de langues ivoiriennes, à tous les niveaux politiques, administratifs, d'enseignements. Dans le domaine public, seuls les médias, les religions, les arts, accordent une modeste place aux langues ivoiriennes. Les langues de grande diffusion sont le français populaire, véhiculaire depuis plus d'un siècle, diffusé partout ; le malinké (groupe mandé) et sa forme véhiculaire appelée dioula ${ }^{2}$ ou dioula tagbusi, répandu lui aussi depuis la colonisation du fait des déplacements nord-sud ; et l'agni et le baoulé, deux langues du groupe kwa très proches, en très bonne position à la fois géographique (sud-est et centre) et politique (durant le $20^{\mathrm{e}}$ siècle). Du fait de leur grande diffusion, certains mots et certaines tournures de ces trois langues sont connus de tous, même de ceux qui ne les parlent pas ou ne les maitrisent pas bien.

8 Le deuxième facteur social en découle : la grande fréquence des pratiques vernaculaires d'alternances de langues où le français est mêlé, en famille et en groupes d'appartenance d'une seule ethnie ou de plusieurs. Par ailleurs, la transmission familiale intergénérationnelle est plus importante pour certaines langues, notamment le malinké et le baoulé, que pour d'autres. L'imprégnation ancienne de ces langues sur le français populaire ivoirien, et la réelle popularité dont jouit le français ivoirien d'aujourd'hui hors des contextes académiques et internationaux amènent les divers milieux professionnels, y compris le milieu enseignant, à la recherche d'une nouvelle norme de français pour la Côte d'Ivoire. Les vernaculaires sont par conséquent composés de ressources de plusieurs langues familiales ou du groupe. Ces ressources sont mutualisées en un fond commun ou pool de ressources fonctionnelles, feature pool (Mufwene 2005 : 99), propice aux influences entre les langues.

9 Un troisième facteur est la gestion par les locuteurs d'une intercompréhension approximative, inévitable en milieu plurilingue. Dans un cadre social où des stratégies de convergence communicationnelle (Giles, Coupland \& Coupland 1991) sont privilégiées, les locuteurs s'accommodent d'une intercompréhension imprécise. Dans une interaction, ils vont d'abord mener une activité cognitive d'adaptation à l'autre, de reformulation de plusieurs façons en modifiant le lexique ou la syntaxe de leurs énoncés pour les rendre proches de la langue de l'autre. Mais lorsqu'un locuteur fait l'effort de parler dans une langue qu'il ne maitrise pas bien, ou lorsqu'un auditeur qui ne maitrise pas bien la langue de l'autre préfère "attribuer un signifié à une chaîne sonore " plutôt que de demander de reformuler, il doit nécessairement pallier la défaillance par une activité de réinterprétation et de révision à l'aide de ses connaissances de la situation ${ }^{3}$ et des probabilités qu'il peut faire : «Pour comprendre l'énoncé que tu entends, suppose que le signifié de la chaîne parlée correspond au type de référent auquel elle semble renvoyer" (Detges 2003 : 55). Joue alors le "principe de référence' (Detges 2003 : 58), cette activité cognitive de réinterprétation pouvant facilement conduire à une recatégorisation des unités.

10 Le quatrième facteur est la créativité continue des locuteurs du nouchi pour manipuler les langues. Le nouchi est l'argot des jeunes Ivoiriens, multiforme, hyper métissé, très répandu dans la société. Thomason (2008) inclut dans les facteurs externes de changement linguistique les manipulations volontaires sur la langue. Or, même s'il est certain que la langue offre peu de résistance au changement lorsqu'elle est volontairement manipulée, comme les manipulations ont surtout une motivation ludique et sont si éphémères, il peut sembler discutable d'intégrer ce facteur dans une théorie générale du changement linguistique. Ce qui se passe avec le nouchi confirme 
l'impact de ce facteur sur une diffusion à partir de la base vers la société haute : alors que les innovations apparaissent dans les ghettos et quartiers précaires des grandes villes, l'engouement de la population ivoirienne et de sa diaspora pour le nouchi ${ }^{4}$ la porte à s'emparer des nouvelles tournures et à leur donner une deuxième vie dans le français ivoirien beaucoup plus stable. Cet état des choses qui perdure depuis plusieurs décennies montre combien ce facteur peut intervenir au cours de l'histoire complexe des situations de contact.

11 À ces facteurs sociaux se mêlent des facteurs plus individuels bien qu'inséparables des contextes sociaux. Comme le disaient déjà Weinreich en 1953 (1967), et Martinet dans la célèbre préface, même si le contact a une dimension essentiellement sociale et collective, il a lieu avant tout chez le locuteur individuel lors de ses discours : «two or more languages will be said to be IN CONTACT if they are used alternately by the same persons. The language-using individuals are thus the locus of the contact. » (Weinreich $1967: 1)$

12 Les mélanges entre les langues du locuteur sont d'autant plus faciles qu'il est exposé à plusieurs langues dès la petite enfance et en acquiert plusieurs de façon informelle durant sa vie du fait de sa mobilité. Ces langues se répartissent dans l'histoire et dans l'aujourd'hui du locuteur selon les espaces sociaux et géographiques. Elles s'organisent aussi par rapport à des modèles de référence, divers pour chacun selon ses instances de socialisation ${ }^{5}$. Par exemple, le standard de France est concurrencé en tant que modèle de prestige du français par un standard de Côte d'Ivoire en cours de construction, tandis que le français populaire ivoirien et le nouchi sont deux modèles de proximité. Pour la langue malinké, un mouvement de recherche de modèles de référence se manifeste depuis quelques années, au profit du koyaka, du mahouka, ou du wojeneka, alors que le modèle de proximité reste le dioula. Ces modèles auront une incidence structurelle sur toutes les langues du locuteur.

13 Au-delà de ces facteurs s'exercent des tendances universelles: processus de véhicularisation (maintien des traits saillants des langues sources, univocité...), dynamique de grammaticalisation vs. dégrammaticalisation, des constantes dans les pratiques vernaculaires (fonctionnalisation, économie, métonymie...), qu'il est sans doute superflu de commenter ici. Il nous semble utile au moins de nous arrêter brièvement sur la convergence des faits observés un peu partout pour le français.

Les variations du français semblent affecter les mêmes zones du système avec une telle régularité que les auteurs distinguent des zones sujettes à variation par rapport à d'autres non sujettes à variation. Le premier exemple de théorisation de cet aspect de la variation est le «français zéro » de Robert Chaudenson, qui remonte à 1981, exposée par lui en 1983 au Colloque de Linguistique et de Philologie romane d'Aix-en-Provence ${ }^{6}$. Dans cette théorie, l'ensemble des variables du français est appelé "français zéro " alors que l'ensemble des traits non sujets à variations, majoritaires, constituent le «noyau dur» de la langue. Lorsque des français "périphériques» hébergent des variantes «hors français zéro », c'est-à-dire affectant une zone de la langue qui n'est atteinte dans aucune autre variété, ces variantes tiennent à des "approximations d'apprentissage » et des processus d'« autorégulation intersystémique ». Les auteurs ajoutent que «l'influence des langues autres que le français [sont] à chercher moins dans des transferts positifs directs (rarissimes) que dans les choix qui s'opèrent (par convergence) entre des variantes des systèmes français et des structures des langues 
des apprenants de ce français " (Chaudenson et al. 1993 : 80). L'importance du locuteur était déjà posée, bien qu'elle ait été peu approfondie dans le cadre du " français zéro ".

Après ces travaux, les lieux de variation du français (Gadet 2011), ou les «tendances inhérentes liées à des dynamiques partagées du français " (Ledegen \& Léglise 2013 : 408) ont été et continuent d'être explorées et commentées. Ces auteures insistent sur le fait que, si les variations sont certainement dues à des facteurs structurels propres au français, elles sont avant tout dues à l'action des locuteurs.

Gadet \& Jones (2008) étudient plusieurs faits de français d'Amérique du nord regardés comme de possibles emprunts grammaticaux liés à une convergence structurelle entre le français et l'anglais. Deux points de leurs conclusions sont à relever ici : d'une part, les faits de changements sont bien plus imputables aux comportements et aux circonstances des locuteurs qu'à des facteurs structurels; d'autre part, ces faits sont plutôt explicables par des principes (probablement universels) jouant sur la manière dont les êtres humains interagissent dans des circonstances ordinaires (Gadet \& Jones $2008: 245)$. Boutin \& Gadet (2012) examinent des convergences et des divergences entre les français d'Afrique et concluent, au regard des points communs entre eux et avec d'autres français, sur la pertinence de regarder la francophonie comme un tout, les français d'Afrique donnant un éclairage utile sur l'ensemble.

17 Ledegen \& Léglise (2013) proposent la notion de résonance pour les aires créolophones, pour exprimer que le français n'est pas seulement influencé par le créole, mais qu'il entre en résonance avec lui, puisque le créole est lui-même un des aboutissements possibles de ses propres tendances (et d'autres). Des faits de français observables lorsqu'il est en contact avec le créole peuvent être observés aussi en Europe, en Amérique et en Afrique, ce qui amène les auteures à constater qu'il existe des «tendances inhérentes liées à des dynamiques partagées du français " (Ledegen \& Léglise 2013 : 408). Les locuteurs du français se rejoignent souvent dans des stratégies hors standard communes, même si chacun des groupes peut considérer comme typiquement à lui telle ou telle des constructions générées, ou encore lui attacher diverses valeurs sociolinguistiques.

18 Devant ces considérations, on peut se demander quelle part reste au contact sur les changements du français. Les recherches sur la part de l'influence des langues en contact et la façon dont elle se développe, notamment dans les français d'Afrique, peinent à s'imposer ou restent toujours un peu à la marge. Un des buts de notre contribution est justement de souligner l'impact du plurilinguisme sur le traitement cognitif des langues par les locuteurs, avec l'avantage que les terrains africains postcoloniaux permettent d'observer en temps réel ce que font les locuteurs avec les langues.

19 Les comportements des locuteurs plurilingues dépassent tout ce qu'un observateur de la langue pourra imaginer après coup. En multipliant les ressources de leur répertoire, le plurilinguisme multiplie l'afflux des possibilités de dire et des façons de dire et, par conséquent, les facteurs de convergence pour qu'une construction soit privilégiée parmi d'autres. Toutefois, l'hétérogénéité est immense et les pratiques ont peu de stabilité (Boutin 2017), au point qu'il est impossible de prédire ce qui, dans la prolifération des créations syntaxiques et lexicales, restera comme résultat des mélanges de codes.

Le comportement prédominant qui se dégage d'abord n'est pas la convergence des langues, mais le fait que les locuteurs acceptent la diversité et le plurilinguisme 
(Canagarajah 2012 : 39) et l'exploitent. Loin de l'idéologie monolingue, ils sont ouverts aux fonctionnements d'autres codes dans le but d'optimiser la communication, et ils assument le contact entre plusieurs langues et styles comme une conséquence immédiate du plurilinguisme qu'ils vivent. La Côte d'Ivoire en est un exemple vivant du fait qu'aucun véhiculaire africain n'a pu s'imposer jusqu'à aujourd'hui et que plusieurs sont en concurrence sur le terrain.

Dans un tel contexte plurilingue, il n'est pas surprenant que les phénomènes et stratégies d'accommodation linguistique et d'ajustement (Giles, Coupland \& Coupland 1991 ; Dragojevic, Gasiorek \& Giles 2016) se développent au niveau pragmatique, et qu'ils donnent lieu un jour à une convergence entre les langues. Toutefois, la position de la langue française est différente dans la mesure où, tout en occupant la première place dans l'officialité, elle ne satisfait pas ses utilisateurs dans sa forme officielle ou de référence ${ }^{7}$. Or lorsqu'une communauté linguistique revendique un droit non seulement à intervenir sur une langue de grande diffusion comme le français, mais à légitimer un nouveau modèle de langue jusque dans ses espaces d'officialité, les langues en contact ne sont pas la seule source pour l'innovation. Des créations peuvent avoir pour source les possibilités même du français. Nous nous proposons de le montrer à travers des exemples de réanalyses, les uns avec convergence et l'autre sans convergence de langues. C'est avec ces exemples que nous développerons les facteurs structurels de convergence.

\section{Les syntagmes prépositionnels avec des fonctions de syntagmes nominaux}

Les facteurs structurels de convergence que nous discutons dans cette section et les suivantes sont l'extension d'emploi d'une construction ou morphème dans une langue par rapport aux emplois de l'équivalent dans les autres langues et la similitude externe de deux constructions dans deux langues (surtout dans les constructions fréquentes). Nous privilégions une optique qui reste proche des pratiques des locuteurs et de ce qu'ils souhaitent faire à travers elles.

L'exemple des constructions adpositionnelles utilisées comme nom, c'est-à-dire assumant les fonctions essentielles des noms, est intéressante dans une discussion sur les convergences sur une même aire plurilingue parce que plusieurs langues à la fois se sont probablement influencées mutuellement (Boutin 2017). Pour partir du français, les énoncés suivants peuvent servir d'exemples de ce type de constructions totalement banal en Côte d'Ivoire :

\begin{tabular}{|l|l|l|}
\hline FIv & (1) & Avant de te moquer du linge de ta voisine, regarde si pour toi est propre (nouchi.com 2/13/01) \\
\hline & (2) & - Docteur, je n'ai pas pu fermer l'œil de la nuit malgré vos conseils \\
\hline & & $\begin{array}{l}\text { - Qui t'a dit de fermer l'œil de la nuit ? au lieu de fermer pour toi c'est pour la nuit que tu veux } \\
\text { fermer ! (http://news.abidjan.net/caricatures/, 2010) }\end{array}$ \\
\hline & $(3)$ & {$[$ à propos d'un commerçant] Je n'achète pas pour lui (oral 04) } \\
\hline & (4) & {$[$ les clignotants] À droite s'allume mais à gauche reste éteint (oral 04) } \\
\hline
\end{tabular}


(5) Ton grand père est toujours là? - Non, il est décédé, c'est chez ma maman qui est encore là (oral 04)

(6) [les bananes] C'est ici qui est mûr, dans la cour n'est pas mûr (oral 98)

E

implicite, comportement langagier courant dans les pratiques vernaculaires. Ce procédé de spécification d'un référent qui n'est pas nommé fait appel à deux stratégies cognitives : l'anaphore et la métonymie. Premièrement, un contexte anaphorique est nécessaire pour qu'un syntagme prépositionnel soit utilisé en discours comme un syntagme nominal à tête vide. Nous entendons ici par anaphore (ang. Anaphora) la procédure qui fait appel à la mémoire immédiate pour identifier un référent déjà manifeste, ou saillant (Kleiber 1994). L'environnement extralinguistique immédiat $(4,6)$ et le discours juste précédent $(1,2,5)$ alimentent la mémoire immédiate, mais il faut compter aussi l'apport des connaissances communes susceptibles d'être convoquées en continuité avec ces deux premières ressources (3). Deuxièmement, la référence ne se fait pas de façon directe, par un pronom spécialisé (ang. Anaphor), mais par une procédure métonymique. Nous appelons métonymie l'établissement d'une relation mentale qui permet de passer d'une entité à une autre qui lui est contigüe (la connectivité comme une des principales facultés de l'esprit humain pour Roman Jakobson). Les prépositions qui entrent dans le type de stratégies discursives étudié ici (spécification d'un référent implicite) sont des prépositions locatives, spécialement aptes à établir des relations de contiguïté. 
Concernant le dioula, l'ordre des constituants place le sujet en début de phrase et les têtes en fin de syntagmes [sujet - objet - verbe] ; le syntagme postpositionnel [nom postposition] est formé avec le même ordre que le syntagme complétif [nom complément - non tête], la postposition ayant la même position qu'un nom tête (Sangaré 1984 inter al.). En dioula, alors que certaines postpositions n'ont qu'une valeur abstraite, l'origine nominale d'autres adpositions reste très proche. Dans ce cas, elles assument aussi une valeur référentielle, comme kóno (dans et ventre), kòro (à côté et dessous, sens), kó (après et dos), bóro (dans, par et main). Une progression dans l'abstraction est visible dans les divers emplois de bóro :

\begin{tabular}{|c|c|c|c|c|c|c|c|}
\hline dioula & (7) & $n$ & bóro & bè & $n$ & & $m i$ \\
\hline & & $1 \mathrm{SG}$ & main & IPFV & $1 \mathrm{SG}$ & \multicolumn{2}{|c|}{ faire mal } \\
\hline & & \multicolumn{6}{|c|}{ (Ma main me fait mal /j'ai mal à la main) } \\
\hline & (8) & $\grave{a}$ & bé & Ala & \multicolumn{3}{|c|}{ bóro } \\
\hline & & $3 \mathrm{SG}$ & COP & Dieu & \multicolumn{3}{|c|}{ main } \\
\hline & & \multicolumn{6}{|c|}{ (C'est dans la main de Dieu/Cela dépend de Dieu) } \\
\hline & (9) & letere & \multicolumn{2}{|c|}{ bé } & \multicolumn{3}{|c|}{ bóro } \\
\hline & & livre & \multicolumn{2}{|c|}{$\mathrm{COP}$} & \multicolumn{3}{|c|}{\begin{tabular}{|l|l|} 
& POSTP
\end{tabular}} \\
\hline & & \multicolumn{6}{|c|}{ (J'ai un livre) } \\
\hline & (10) & \multicolumn{2}{|l|}{ derèké } & \multicolumn{2}{|l|}{ fara-la } & yàó & bóro \\
\hline & & \multicolumn{2}{|l|}{ chemise } & \multicolumn{2}{|c|}{ déchirer-PFV } & Yao & POSTP \\
\hline & & \multicolumn{6}{|c|}{ (La chemise a été déchirée par Yao) } \\
\hline
\end{tabular}

En (7) et (8), bóro est un nom, susceptible de recevoir des marques de spécification, tête de syntagme. Il est vrai qu'une phrase comme (8) issue de la Bible permet déjà de percevoir un sens plus abstrait à l'expression bé $X$ bóro (dans la main de X). En (9) et (10), bóro est une postposition, tête d'un syntagme adpositionnel, obligatoire en (9) et facultatif en (10).

En dioula, il existe une grande facilité pour que des éléments adverbiaux (équivalents de ici, demain...) et des syntagmes adpositionnels occupent des positions nominales. De la sorte, l'énoncé (6) du français ivoirien a son exact équivalent en dioula. Le baoulé permet la même construction, contrairement aux pratiques classiques du français :

\begin{tabular}{|l|l|l|l|l|l|l|l|l|}
\hline dioula & $(11)$ & yàn & lé & mònnan, & bo & kónon & man & mòn \\
\hline & & ici & COP & mûr & maison & dans & COP.NEG & mûr \\
\hline
\end{tabular}




\begin{tabular}{|l|l|l|l|l|l|l|}
\hline baoulé & $(12)$ & wàfan & lo-li, & àwlo & nun & wa-lo-man \\
\hline & & ici & mûrir-PFV & cour & dans & AUX-mûr-NEG \\
\hline & & (FIv : Ici est mûr, dans la cour n'est pas mûr) \\
\hline & (Celles d'ici sont mûres, celles de/dans la cour ne sont pas mûres) \\
\hline
\end{tabular}

En baoulé, l'ordre des constituants est [sujet - verbe - objet], mais dans les syntagmes complétifs, l'ordre est [nom complément - non tête]. Les éléments qui introduisent un syntagme nominal pour former un syntagme de rang supérieur, circonstant ou de complément d'un nom ou d'un verbe, ne constituent pas une classe d'adpositions (Creissels et Kouadio 1977: 347-354). S'ils sont d'origine nominale, ils conservent certaines de leurs propriétés, et le syntagme complétif formé est apte à occuper toutes les fonctions nominales dans la phrase. La langue utilise, dans un but de localisation, des noms relationnels, par exemple de parties du corps, dont le sens peut évoquer une localisation plus générale (Creissels et Kouadio, 1977: 354-365) : sú (surface), nun (intérieur), nyrun (visage), sin (dos), àfien (milieu, taille), etc. Dans une traduction française, les équivalents sémantiques (mais non syntaxiques) suivants peuvent être donnés : sú sur; nun dans; nyrun devant; sin derrière; àfien au milieu de. Afien nous servira à montrer les propriétés de ces items.

\begin{tabular}{|c|c|c|c|c|c|c|c|c|}
\hline baoulé & (13) & \multicolumn{2}{|l|}{ bian } & $n g a$ & $\boldsymbol{i}$ & àfien & \multicolumn{2}{|c|}{ cEnnin } \\
\hline & & \multicolumn{2}{|l|}{ homme } & DEM & $3 S G$ & tronc & \multicolumn{2}{|c|}{ être grand } \\
\hline & & \multicolumn{7}{|c|}{ (Cet homme a un gros tronc) } \\
\hline & (14) & $i$ & \multicolumn{2}{|l|}{ suà } & wò & klo & $i$ & àfien \\
\hline & & $3 S G$ & \multicolumn{2}{|c|}{ maison } & être & village & $3 S G$ & milieu \\
\hline & & \multicolumn{7}{|c|}{ (Sa maison est au milieu du village) (Creissels et Kouadio $1977: 361-362$ ) } \\
\hline & (15) & \multicolumn{2}{|l|}{$\boldsymbol{\jmath}$} & wò & be & \multicolumn{3}{|l|}{ àfien } \\
\hline & & \multicolumn{2}{|l|}{ 3SG.NOM } & être & $3 \mathrm{PL}$ & \multicolumn{3}{|l|}{ milieu } \\
\hline & & \multicolumn{7}{|c|}{ (Il est parmi eux) } \\
\hline
\end{tabular}

31 Be et $\boldsymbol{i}$ ne sont pas des déterminants, mais des pronoms ( $\boldsymbol{i}$ ne peut pas être sujet); antéposés à àfien, ils ont une fonction de complément de àfien qui reste un nom, avec une valeur plus abstraite dans (14) et (15). Le baoulé a la possibilité de positionner ce type de syntagmes en sujet:

\begin{tabular}{|l|l|l|l|l|l|}
\hline baoulé & $(16)$ & bé & àfien & ti & saciwa \\
\hline
\end{tabular}




\begin{tabular}{|l|l|l|l|l|l|}
\hline & & 3PL & milieu & être & détérioré \\
\hline & & \multicolumn{4}{|c|}{ (Les relations entre eux sont rompues) (Tymian et al. 2003: 60) } \\
\hline
\end{tabular}

Tymian et al. (2003) propose, dans un tel cas, une sémantisation de àfien en « relation ». Le français ivoirien, de son côté, n'hésite pas à produire en équivalent de (16) la phrase :

\begin{tabular}{|l|l|l|}
\hline FIv & $(17)$ & Entre eux est gâté. \\
\hline
\end{tabular}

De la même façon, toutes les phrases (1) à (6) sont directement traduisibles en baoulé sans aucun pronom de reprise.

Alors que le français possède des prépositions, qui forment dans une catégorie totalement distincte des noms même si elles dérivent souvent de noms relationnels, le baoulé, à l'instar des autres langues akan, ne possède pas d'adpositions. Le baoulé utilise les noms relationnels susceptibles de fournir une localisation avec leur complément de nom antéposé, lorsque le français, à l'instar des langues romanes, utilise des syntagmes prépositionnels locatifs. Le dioula représente un cas intermédiaire puisqu'il possède des postpositions formelles qui n'ont aucune propriété nominale, et des postpositions locatives, de même forme et de même construction que des noms relationnels, mais n'ayant plus les propriétés de spécification des noms. Ces trois langues pourraient détailler l'échelle de grammaticalisation des noms en adpositions de Hopper \& Traugott (2003: 110-111) dans un processus convergent, sans que l'on puisse encore dire si le changement sera accéléré, pour le baoulé par exemple, $\mathrm{du}$ fait du contact. En revanche, les locuteurs du français ivoirien qui connaissent les propriétés de langues comme le dioula ou le baoulé, accordent facilement des propriétés nominales à des syntagmes prépositionnels locatifs, même si les prépositions n'ont pas une origine nominale comme entre (16) ou dans (6), semblant ainsi aller à l'inverse du processus.

\section{Le cas de pour (N/PRO) ; (N/PRO) lic̀ (baoulé) et (N/PRO) tá (dioula)}

Pour (N/PRO), tout en étant un des syntagmes adpositionnels utilisés avec des propriétés nominales comme les précédents, mérite d'être traité à part, étant donné la complexité de cette préposition en français, étudiée minutieusement par Cadiot (1991). Pour (N/PRO) est sans doute un des cas où la convergence des langues se manifeste le mieux.

Dans les exemples $(1,2,3)$, pour ( $N / P R O)$ est totalement intégré syntaxiquement et aucune dislocation n'est possible, ni aucune reprise anaphorique n'est nécessaire. Pour (N/PRO) fait partie de l'Enoncé-Champ-d'Incidence de Cadiot (1991), contrairement aux cas que l'auteur étudie, où « le contenu de POUR $\Omega$ a le statut d'un fait acquis sur lequel c'est l'Enoncé-Champ-d'Incidence qui enchaine. [...] le contenu de POUR $\Omega$ est représenté comme ayant des fondements énonciatifs extérieurs à ceux de la présente énonciation, celle du locuteur de l'ECI. » (Cadiot $1991: 25)^{9}$ 
37 Le cas du pour (N/PRO) spécifique au français ivoirien est, en revanche, comparable à celui de (N/PRO) lic̀ (baoulé) et de (N/PRO) tá (dioula), dont les propriétés systémiques sont décrites dans Creissels \& Kouadio (1977 : 327-341) et Sangaré (1984: 280-289). Un examen précis des propriétés de pour, de lic̀ et de tá amène à la conclusion qu'aucun des trois morphèmes n'a toutes les propriétés d'un autre, bien qu'une influence mutuelle entre les trois soit très probable ${ }^{10}$.

En dioula actuel, la postposition tá (contrairement à pour en français) forme des syntagmes génitifs du type : Awa tá mobili (la voiture de/*pour Awa) dans le cas de possessions aliénables. Lors d'une réduction discursive de ces syntagmes, tá forme la tête du syntagme postpositionnel : Awa tá (celui/celle de Awa). Ce syntagme postpositionnel repère un référent implicite et fonctionne comme un sujet ou un complément dans les exemples qui suivent, (19) correspondant à (3) du français ivoirien.

\begin{tabular}{|c|c|c|c|c|c|c|c|}
\hline dioula & (18) & Awa & $t a ́$ & bé & \multicolumn{3}{|c|}{ yèn } \\
\hline & & Awa & POSTP & être & \multicolumn{3}{|c|}{$i c i$} \\
\hline & & \multicolumn{6}{|c|}{ (FIv : Pour Awa est ici) } \\
\hline & & \multicolumn{6}{|c|}{ ([la voiture] Celle de Awa est ici/ Il y a celle de Awa ici) } \\
\hline & (19) & $n$ & $t \varepsilon ́$ & $\grave{a}$ & & tá & sàn \\
\hline & & $1 \mathrm{SG}$ & IPFV.NEC & & & POSTP & acheter \\
\hline & & \multicolumn{6}{|c|}{ (FIv : Je n'achète pas pour lui) } \\
\hline & & \multicolumn{6}{|c|}{ (Je n'achète pas sa marchandise) } \\
\hline
\end{tabular}

Il est intéressant de remarquer qu'une réanalyse du bambara a eu lieu en dioula. En bambara, la possession aliénable est marquée par ká postposé au possesseur: $\boldsymbol{n}$ ká mobili (ma voiture) ; la relation inaliénable n'est marquée par aucune postposition : $\boldsymbol{n}$ denmusó (ma fille). Tá du dioula est une refonctionnalisation en marqueur de possession aliénable d'un morphème focalisateur du possesseur en bambara (Sangaré 1984 : 280-289). En bambara tá, qui est à l'origine un nom, focalise le possesseur dans $\boldsymbol{n}$ tá denmusó («ma fille à moi ») par rapport à $n$ denmusó (ma fille). En dioula actuel de Côte d'Ivoire, $k a ́$ est le plus souvent remplacé par tá, réanalysé comme une postposition marquant la possession aliénable : $\boldsymbol{n}$ tá mobili (ma voiture) et pouvant former un syntagme postpositionnel autonome: $\boldsymbol{n}$ tá (le mien/ la mienne/ les miens/ les miennes).

Peut-être pendant le même temps, pour a suivi une évolution parallèle (mais non analogue). Au tout début du $20^{e}$ siècle dans l'ancienne AOF, pour entrait dans des constructions complétives du type : case pour lui / case pour nous / case pour vous / case pour eux ("sa maison/ notre maison/ votre maison/ leur maison»). Ces constructions génitives étaient données comme exemples dans des documents militaires de France destinés à l'enseignement du français aux tirailleurs, comme Le français tel que le parlent nos tirailleurs sénégalais (Anonyme 1916: 9). Pour n'est plus utilisé depuis des décennies 
dans de tels syntagmes génitifs, mais le français ivoirien a ce pour tête de syntagmes prépositionnels qui repèrent, comme en (1-3), un référent implicite et fonctionnent comme des noms en discours (pour lui est fermé/ le sien est fermé (2)). Toutefois, l'élément implicite ne résulte pas d'une ellipse, pour la raison qu'il n'est pas réalisable aujourd'hui dans ces structures. Il semble donc que pour ait premièrement été refonctionnalisé dans le français tirailleur ${ }^{11}$ comme préposition d'appartenance précédant le possesseur, peut-être à partir de constructions attributives telles que : c'est pour lui. Il a ensuite formé des syntagmes autonomes pour (N/PRO), sans doute au contact du baoulé lors de la période coloniale.

41 Le baoulé possède le nom relationnel lì̀ de sens " part, chose ", qui est toujours la tête de syntagmes génitifs, son complément du nom lui étant antéposé. En particulier, il entre dans des énoncés du type de $(1,2,3)$ comme le montrent (22-23).

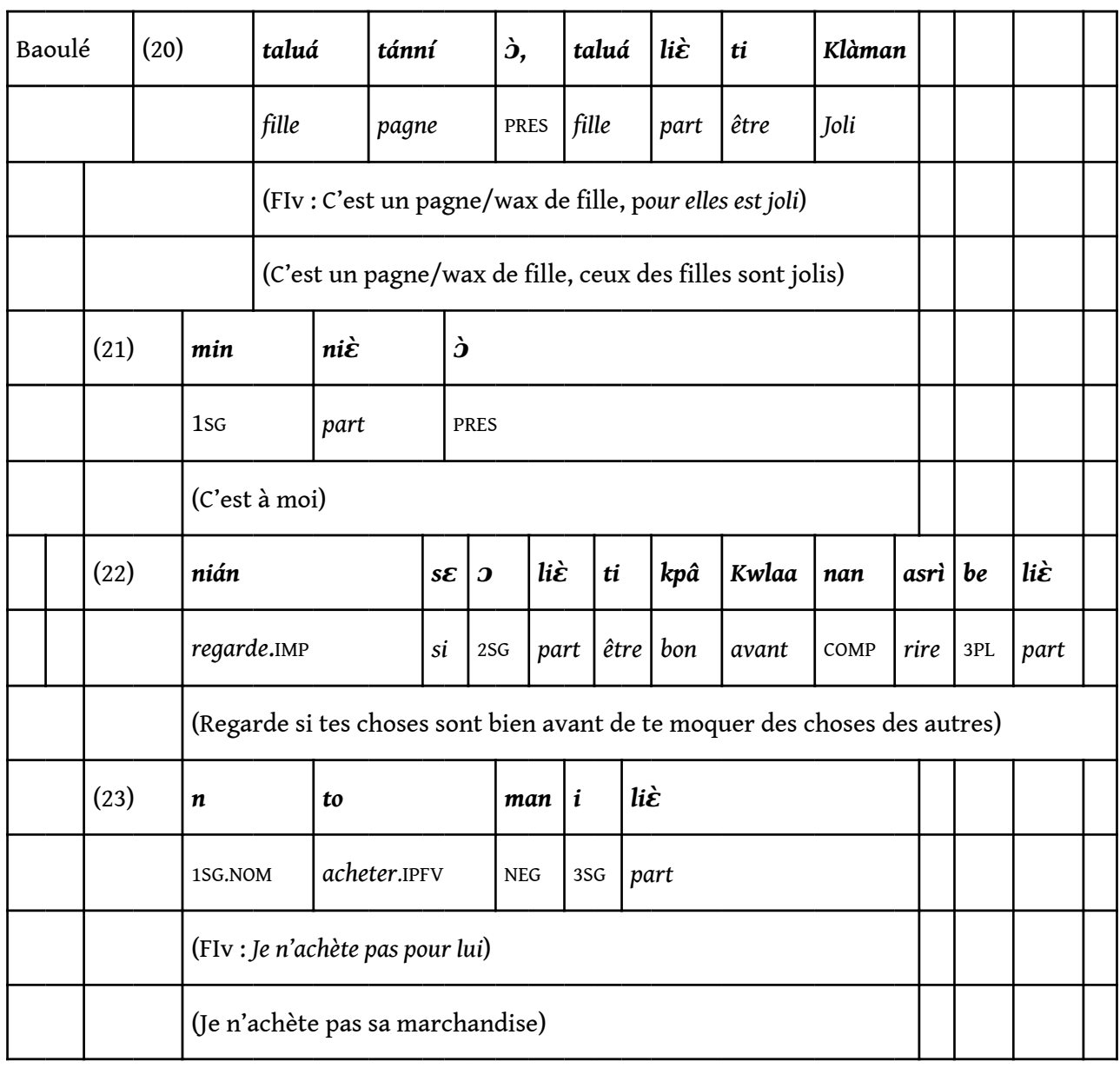

Comme le pour actuel du français ivoirien, mais contrairement à tá du dioula dans Awa tá mobili (la voiture de Awa) et à l'ancien pour du français tirailleur (case pour lui), liè n'est jamais utilisé dans des syntagmes génitifs complément d'une tête nominale. En revanche, il est un nom tête de syntagmes génitifs et peut recevoir des marques de spécification, au contraire de pour.

Par ailleurs, comme pour en français, mais non tá en dioula, liè entre dans des syntagmes détachés en tête d'énoncé pour la thématisation du sujet : 


\begin{tabular}{|l|l|l|l|l|l|l|l|l|}
\hline baoulé & $(24)$ & wó & lì̀, & $\boldsymbol{a}$ & $\boldsymbol{k}$ wlá & wanndì & trà & $\boldsymbol{m i n}$ \\
\hline & & 2SG & POSTP & 2SG.NOM & Pouvoir.IPFV & courir & dépasser & $1 \mathrm{SG}$ \\
\hline & & \multicolumn{6}{|c|}{ (Quant à toi /Pour toi, tu cours plus vite que moi) } \\
\hline
\end{tabular}

Les trois morphèmes liè, tá et pour partagent certaines propriétés l'un avec l'autre, mais ils sont tous d'origine très différentes et aucun n'est totalement équivalent à l'autre. Comme les utilisations de pour se sont étendues aux premiers temps du contact avec le manding d'abord, puis du contact avec le baoulé, il est très probable que les emplois de tá et de liè aient été étendus aussi au contact du français. Au siècle dernier, sur la même aire communicative, plusieurs processus convergents ont eu lieu. Tá a perdu ses propriétés nominales en dioula tagbusi, dans le même temps où pour convergeait avec lui dans des emplois comme préposition dans des syntagmes génitifs. Puis pour a acquis des emplois comme tête de syntagmes prépositionnels référentiels, à l'instar de liè. Liè est resté un nom en baoulé et est utilisé en fonction de sujet et complément, mais perd ses propriétés nominales lorsqu'il est utilisé pour une thématisation détachée (24).

\section{Un conditionnel périphrastique en français}

Le conditionnel périphrastique ${ }^{12}$ est bien attesté en Côte d'Ivoire et au Burkina Faso, dans une moindre mesure au Mali, conjointement au conditionnel classique. Il est un exemple de combinaison originale à partir de réanalyses de sources sémantiques et formelles diverses, mais l'hypothèse d'une convergence des langues ne peut être validée. Sa construction s'intègre dans les procédés des périphrases verbales, bien attestées pour le français à la période classique (Fournier $1998: 262-264$ ), ainsi que pour les dialectes proches du français à partir du $17^{e}$ (Chaudenson et al. 1993 : 81-97). Les exemples de parole spontanée dans cette section sont tirés de trois corpus construits entre 2004 et 2011 à Abidjan : PFC «Phonologie du français contemporain » (https:// www.projet-pfc.net/), CFA "Contemporary French in Africa " (Université d'Oslo) et CIEL-F « Corpus international écologique de langue française » (http://ciel-f.org) ${ }^{13}$.

Les formes périphrastiques concurrencent les formes synthétiques des temps verbaux du français, du fait de leur meilleure maniabilité et de leur plus grande transparence sémantique. En effet, d'une part, la conjugaison d'un seul auxiliaire ou semi-auxiliaire remplace la conjugaison (souvent irrégulière) d'un grand nombre de verbes et, d'autre part, l'information sémantique du temps, mode ou aspect verbal apparait dans un morphème séparé du verbe, tandis que le verbe, sous une forme unique, donne l'information sémantique lexicale (un mot correspond alors à une information). Cette forme unique du verbe est traditionnellement perçue comme l'infinitif, mais au niveau phonologique, l'infinitif et le participe passé se distinguent rarement. Par exemple, dans tous les espaces francophones, mangé/ manger et marché/marcher se prononcent respectivement [mãze] et [ma: $[\mathrm{e}] /\left[\mathrm{maB} \int \mathrm{e}\right]$, mais dans les aires où les $\mathrm{R}$ finaux sont élidés, comme en Côte d'Ivoire, parti/partir et fini/finir se prononcent de la même façon : [pa:ti] et [fini] ${ }^{14}$. 
47 Le conditionnel périphrastique est une forme analytique en aller à l'imparfait suivi du verbe à l'infinitif. Il apparait par exemple dans des discours fictionnels hypothétiques, après une subordonnée en si à l'imparfait (ou au plus que parfait) comme :

\begin{tabular}{|l|l|}
\hline $\begin{array}{l}\text { CI/ } \\
\text { BF }\end{array}$ & \begin{tabular}{l} 
(25) Si on partait en huitième de finale [...] on allait arriver (CIEL-CI-F2, 2010) \\
\hline
\end{tabular} \\
\hline $\begin{array}{l}\text { (26) Si j'étais riche sincèrement j'allais aider ceux qui sont dans le besoin (CFA-CI, 2008) } \\
\text { (CFA-CI-2008) }\end{array}$ \\
\hline & (28) Si on avait pu déplacer tous les Gurunsi là ça allait faire de la place (PFC-BF, 2004) \\
\hline
\end{tabular}

48 Cette flexion, que Boutin \& Kouamé (2011) avons appelée conditionnel périphrastique, s'appuie sur une réanalyse à partir des valeurs déjà associées à l'imparfait en français. Le conditionnel périphrastique en allait INFINITIF apporte l'innovation d'une valeur pour cette forme et d'une correspondance de plus entre flexion synthétique et flexion analytique. En effet, au conditionnel synthétique classique correspond désormais le conditionnel périphrastique.

49 La forme allait INFINITIF existe en français de référence pour une action du passé, proche d'avoir été réalisée. Cependant, dans une structure hypothétique en français de référence, alors que le futur périphrastique en aller peut facilement remplacer le futur simple, la périphrase en aller ne peut pas remplacer efficacement le conditionnel dans la principale :

\begin{tabular}{|l|l|l|}
\hline F référence & $(29 \mathrm{a})$ & Si tu as de l'argent, tu vas le dépenser \\
\hline & $(29 \mathrm{~b})$ & Si j'avais de l'argent, j'allais le dépenser \\
\hline
\end{tabular}

50 Une valeur conditionnelle pour (29b) est peu accessible en français de référence : allais est plus facilement interprété comme un verbe de mouvement que comme un auxiliaire d'aspect, avec une valeur itérative. Sans le conditionnel (simple) dans la principale, si peut être substituable par quand en français de référence et une hypothèse ne peut pas automatiquement être formée.

51 L'auxiliation en aller en français de référence est aspectuelle : aller suivi d'un verbe à l'infinitif porte une valeur d'imminence (futur proche), avec éventuellement une visée prospective ou intentionnelle. C'est probablement cette valeur intentionnelle de aller qui est exploitée pour former (parmi d'autres emplois) notre conditionnel périphrastique, utilisé dans des discours fictionnels hypothétiques tels que (25) à (28). Par ailleurs, l'imparfait dénote un procès situé hors de l'actualité, soit avec une valeur temporelle de passé, soit avec une valeur d'irréel, ou contrefactuelle (Berthonneau \& Kleiber 2006). Dans le français ouest-africain, ce sont ces deux valeurs existantes en français actuel qui sont mises en avant: l'auxiliaire aller, qui devient le support de l'imparfait, et lui permet une valeur intentionnelle ou prospective dans l'expression de l'éventualité (Boutin \& Kouamé 2011). 
On peut remarquer en (29), en contraste, l'imparfait utilisé avec sa valeur irréelle mais sans auxiliaire aller, donc sans valeur d'intentionnalité dans cette plainte d'une institutrice :

(29) [le nouchi] si on le parlait simplement comme ça pour jouer, c'était encore mieux, mais, ça se répercute sur les écrits (PFC-CI, 2005)

On peut aussi confirmer la valeur intentionnelle de aller, à l'imparfait donc déconnectée de la réalité, dans des énoncés comme (30), où un gestionnaire d'une association solidaire rapporte les mauvaises intentions qu'on lui a prêtées :

(30) Mais Madame pour qu'on puisse trouver les fonds nécessaires pour faire venir les trois blocs opératoires j'ai marché pendant trois mois [claquements de mains] comme si j'allais emmener pour vendre ou bien emmener $d / d$ /dans mon village (PFC-BF, 2004)

54 Nous avions recherché (Boutin \& Kouamé 2011) dans quelle mesure le baoulé pouvait influencer la réanalyse de la forme allait INFINITIF chez les locuteurs bilingues baoulé français à l'aide du corpus de l'enquête $\mathrm{CFA}-\mathrm{CI}$, qui les enregistre à la fois en baoulé et en français sur des tournures hypothétiques. Nos analyses concluent que seule une influence sémantique est possible, puisque le baoulé possède un mode intentionnel (avec une réalisation du verbe sur un ton haut). Les locuteurs de CFA-CI utilisent effectivement le conditionnel périphrastique lorsqu'ils s'expriment en français et, de façon équivalente, le mode intentionnel lorsqu'ils s'expriment en baoulé. Le baoulé a bien un auxiliaire wa ("venir ») qui peut avoir soit une valeur future, soit une valeur intentionnelle; celui-ci apparait une seule fois dans le corpus. Toutefois, dans un discours hypothétique, l'ajout du mode intentionnel à wa est obligatoire pour que l'hypothèse sorte du réel (Voir Boutin \& Kouamé 2011). On ne peut donc pas conclure à une influence $\mathrm{du}$ baoulé concernant l'auxiliation avec aller du conditionnel périphrastique.

Le mode intentionnel existe dans plusieurs langues de Côte d'Ivoire à l'instar du baoulé. Cette modalité n'apparaissant pas du tout dans le conditionnel synthétique du français actuel, le besoin a pu se faire sentir d'une nouvelle forme exprimant cette valeur en Côte d'Ivoire. Toutefois, dans l'état actuel de nos recherches, nous ne pouvons pas déterminer si l'utilisation du mode intentionnel dans des pays sahéliens vient d'une influence de certaines langues ou plutôt s'il s'est répandu à partir du français de Côte d'Ivoire. Skattum (2011), qui analyse les éventuelles interférences du bambara ou du dioula dans le français du Mali et de la Côte d'Ivoire, montre qu'on ne peut pas conclure à une influence de ces langues sur le conditionnel périphrastique. Tous ces énoncés sont interprétables par la majorité des francophones d'Afrique et d'ailleurs, mais ils ne sont pas attestés dans tous les français. Au contraire, le conditionnel synthétique est souvent étendu à la subordonnée après si dans les français européens (si j'aurais...), ce qui n'est pas du tout répandu dans l'aire ouest-africaine.

56 Le conditionnel périphrastique illustre un cas de réanalyse sémantique d'une forme verbale pour en faire une nouvelle catégorie modale à partir des possibilités même du 
français, mais l'hypothèse d'une convergence structurelle avec les langues en contact semble à écarter.

\section{Conclusion}

57 Suite à la journée d'étude Sociétés plurilingues et contact de langues : des descriptions aux réflexions épistémologiques du 11 janvier 2016 à Yaoundé, ma contribution écrite avait porté sur les influences mutuelles des langues en contact en Côte d'Ivoire (Boutin 2017). Le but était de rappeler la multiplicité des phénomènes (alternances, métissages, emprunts, interférences et convergences divers), et la variabilité de l'influence d'une langue sur une autre. J'avais insisté sur le fait que des facteurs structurels, individuels et sociaux se mêlent non seulement dans les interférences dues aux contacts, mais bien avant dans les pratiques langagières. Suite aux journées d'étude La convergence des 18-19 octobre 2019 à Aix-en-Provence, la réflexion a continué avec l'observation des facteurs convergents sur quelques exemples de constructions.

Les langues de plus grande diffusion, en l'occurrence le français ivoirien, le dioula et le baoulé se sont modifiées mutuellement du fait de plusieurs facteurs tels que la large connaissance de leurs principales constructions, la façon dont les locuteurs gèrent une intercompréhension approximative par la tolérance formelle et la reformulation diversifiée, la popularité d'un argot métissé et les pratiques plus ou moins consensuelles d'alternances de langues. C'est en se servant de leur répertoire et de leur compétence plurilingues que les locuteurs ont opéré des réanalyses ou des adaptations (Gumperz 1964, Gumperz \& Wilson 1971) par convergence entre les fonctionnements des langues en présence.

Alors que le baoulé, le dioula et le français représentent, dans l'hypothèse de l'échelle de grammaticalisation des noms en adpositions de Hopper \& Traugott (2003:110-111), des degrés distincts, on constate que le français ivoirien a acquis des traits qui remettent en cause la directionnalité même du processus. Concernant les convergences et les divergences de pour en français, de tá en dioula et de liè en baoulé, la comparaison avec un français véhiculaire ancien dont on possède des sources écrites, et avec des langues manding n'ayant pas connu la véhicularisation et le contact qu'a connu le dioula, met en évidence des processus divers de réanalyses faisant converger les propriétés de ces morphèmes.

60 En revanche, il n'est pas possible de trouver au conditionnel périphrastique attesté en Afrique de l'ouest, créé à partir de plusieurs réanalyses de l'auxiliaire aller et des temps verbaux du français, un modèle formel dans les langues en contact. Pourtant, alors qu'aucune convergence structurelle n'est visible, une influence des langues en contact est possible à un niveau sémantique plus profond du fait du plurilinguisme des locuteurs : les facteurs extralinguistiques rappelés ci-dessus convergent pour favoriser de telles réanalyses. La notion de convergence ne s'avère pas toujours discriminante dans le cas d'une multiplicité de facteurs susceptibles de se joindre à des niveaux différents pour occasionner le changement linguistique. Elle a besoin d'être ancrée dans la prise en compte beaucoup plus large de l'écologie linguistique, telle que l'approchent par exemple Ludwig, Pagel \& Mühlhäusler (2018), comme un tout sociohistorico-culturel, avec des déterminants biologiques. Le modèle de l'écologie linguistique que présentent ces auteurs, ou celui de Mufwene (2008), qui tentent de 
montrer comment ces multiples facteurs interagissent, sont sans doute de meilleurs cadres pour aller plus loin dans l'analyse.

\section{BIBLIOGRAPHIE}

Berthonneau, A-M. \& G. Kleiber (2006) Sur l'imparfait contrefactuel, Travaux de linguistique, 53, p. 7-65.

Boutin, A.B. (2017) A propos de quelques influences mutuelles des langues en contact en Côte d'Ivoire, Cahiers de linguistique : Sociétés plurilingues et contact de langues: des descriptions linguistiques aux réflexions épistémologiques, 43, 2, p. 11-33.

Boutin, A.B. \& K. Kouamé (2011) Analyse énonciative comparée des systèmes hypothétiques en si en français de Côte d'Ivoire et en se en baoulé dans un discours fictionnel, in Boutin, A.B. (ed.) Autour du verbe, Le français en Afrique, 26, Nice: ILF, CNRS, p. 71-84.

Boutin, A.B. \& F. Gadet (2012) Comment ce que montrent les français d'Afrique s'inscrit/ne s'inscrit pas dans les dynamiques des français dans une perspective de francophonie, in Blumenthal, P. \& S. Pfänder (eds.) Convergences, divergences et la question de la norme en Afrique francophone, Le français en Afrique, 27, Nice: ILF, CNRS, p. 19-34.

Boutin, A.B. \& J. Kouadio N'Guessan (2015) «Le nouchi c'est notre créole en quelque sorte, qui est parlé par presque toute la Côte d'Ivoire ", in Blumenthal, P. (ed.), Dynamique des français africains : entre le culturel et le linguistique, Bern: Peter Lang, p. 251-271.

Cadiot, P. (1991) De la grammaire à la cognition : la préposition pour, Paris: CNRS Editions.

Canagarajah, A.S. (2012) Translingual Practice: Global Englishes and Cosmopolitan Relations. New York: Routledge.

Chaudenson, R., Mougeon, R. \& E. Béniak (1993) Vers une approche panlectale de la variation du français, Paris: Didier Erudition.

Creissels, D. \& J. Kouadio N'Guessan (1977) Description phonologique et grammaticale d'un parler baoulé, Abidjan: ILA.

Detges, U. (2003) La notion de réanalyse et son application à la description des créoles français, in Kriegel, S. (ed.), Grammaticalisation et réanalyse. Approches de la variation créole et française, Paris: CNRS Editions, p. 49-67.

Dragojevic, M., Gasiorek, J., \& H. Giles (2016) Accommodative Strategies as Core of the Theory, in Giles, H. (ed.) Communication Accommodation Theory: Negotiating Personal Relationships and Social Identities across Contexts, Cambridge, MA: CUP, p. 36-59.

Ebongue, A.E. \& E. Hurst (eds.) (2017) Sociolinguistics in African Contexts: Perspectives and Challenges, Springer International Publisher (Multilingual Education, 20).

Fournier, N. (1998) Grammaire du français classique, Paris: Belin Sup-Lettres. 
Gadet, F. (2011) La palette variationnelle des français, in Martineau, F. \& T. Nadasdi (dirs.) Le français en contact. Hommages à Raymond Mougeon, Québec: Presses de l'université de Laval, p. 117-148

Gadet, F. \& M.C. Jones (2008) Variation, Contact and Convergence in French Spoken outside France, Journal of language contact, Thema 2, p. 238-248.

Giles, H., Coupland, N., \& J. Coupland (1991) Accommodation theory: Communication, context, and consequence, in Giles, H., Coupland, J. \& N. Coupland (eds.) Studies in emotion and social interaction. Contexts of accommodation: Developments in applied sociolinguistics, New York: Cambridge University Press, p. 1-68.

Gumperz, J.J. (1964) Linguistic and Social Interaction in Two Communities, in Gumperz, J.J. \& D. Hymes (eds.) The Ethnography of Communication. American Anthropologist, 66, 6, II (Special Issue), p. 137-153.

Gumperz, J.J. \& R. Wilson (1971) Convergence and Creolization: A Case From the Indo-Aryan/ Dravidian Border in India, in Hymes, D.H. (ed.) Pidginization and Creolization of Languages, London: Cambridge University Press, p.151-167. [Repris dans Gumperz, J.J. (1971) Language in Social Groups. Essays, in Dil A.S. (ed.), Stanford: Stanford University Press, p. 251-273]

Hopper, P.J. \& E.C. Traugott (2003) Grammaticalization, Cambridge: Cambridge University Press.

Kleiber, G. (1994) Anaphores et pronoms, Louvain-la-Neuve: Duculot.

Kriegel, S. (2003) Introduction : Vers une interprétation multicausale du changement linguistique, in Kriegel, S. (ed.) Grammaticalisation et réanalyse. Approches de la variation créole et française, Paris: CNRS Editions, p. 3-37.

Kriegel, S. (2018) La convergence à l'origine de nouvelles langues, Séminaire de l'équipe SYSTUS, 19 janvier 2018, LPL, Aix-en-Provence. Disponible sur : https://amupod.univ-amu.fr/video/1464seminaire-du-laboratoire-parole-et-langage-cnrs-amu-19-janvier-2018-equipe-de-recherchesystus-systemes-et-usages/

Anonyme (1916) Le français tel que le parlent nos tirailleurs sénégalais, Paris: Imprimerie Militaire Universelle L. Fournier.

Ledegen, G. \& I. Léglise (2013) Variation et changements linguistique, in Simonin, J. \& S. Wharton (dirs) Sociolinguistique du contact. Dictionnaire des termes et concepts, 15, Lyon: ENS Editions, p. 399-418.

Léglise, I. (2018) Pratiques langagières plurilingues et frontières de langues, in Auzanneau, M. \& L. Greco (eds.) Dessiner les frontières, Lyon: ENS Editions, p. 143-169.

Ludwig, R., Pagel, S. \& P. Mühlhäusler (2018) Linguistic Ecology and Language Contact, Cambridge: Cambridge University Press.

Mufwene, S.S. (2005) Créoles, écologie sociale, évolution linguistique, Paris: L'Harmattan (Langues et Développement).

Mufwene, S.S. (2008) Language Evolution: Contact, Competition and Change, London. New York: Continuum International Publishing Group Ltd.

Nunez J.J.F. \& I. Léglise (2016) Ce que les pratiques langagières plurilingues au Sénégal disent à la linguistique du contact, in Auzanneau, M., Bento, M. \& M. Leclère (dirs.) Espaces, mobilités et éducation plurilingues : Éclairages d'Afrique ou d'ailleurs, 7, Paris: Éditions des Archives contemporaines, p. 99-119. 
Sangaré, A. (1984) Dioula de Kong (Côte d'Ivoire) : phonologie, grammaire, lexique et textes, thèse de 3e cycle, Université Grenoble 3.

Skattum, I. (2011) Si j'étais riche... Constructions hypothétiques en français parlé au Mali, in Boutin A.B. (ed.) Autour du verbe, Le français en Afrique, 26, Nice: ILF, CNRS, p. 49-70.

Tymian, J., Kouadio N'Guessan J. \& J.-N. Loucou (2003) Dictionnaire baoulé français, Abidjan: Nouvelles éditions Ivoiriennes.

Thomason, S.G. (2008) Social and Linguistic Factors as Predictors of Contact-Induced Change, Journal of Language Contact, Thema 2: Language Contact and the Dynamics of Language / Theory and Implications, p. 42-56.

Weinreich, U. (1967) Languages in contact. Findings and Problems. (1 ${ }^{\text {re }}$ éd. 1953) The Hague: Mouton \& Co.

Winford, D. (2003) An Introduction to Contact Linguistics, Oxford: Blackwell.

\section{NOTES}

1. Je remercie Médéric Gasquet-Cyrus et un relecteur anonyme pour leurs remarques constructives. Ils ne sont pas responsables des défauts de clarté qui ont pu rester dans ce texte.

2. Le dioula est plus proche du bambara parlé au Mali que du malinké de Côte d'Ivoire. Le terme dioula désigne le commerçant en bambara (à bé julaya ké. 3SG IPFV commerce faire. Il fait du commerce). Avant la colonisation, le dioula est parlé au Nord de la Côte d'Ivoire actuelle, dans le royaume Kong.

3. Nous entendons ici par situation non seulement l'environnement immédiat, mais aussi les connaissances acquises des locuteurs, leurs gestes, et les enjeux communicationnels de l'interaction.

4. Il ne s'agit pas seulement de la population ivoirienne mais aussi de celle de l'Afrique de l'Ouest et au-delà de la France où des mots nouchi entrent peu à peu dans le Robert.

5. Nous entendons ici par socialisation le processus d'intériorisation des normes et des valeurs des groupes d'appartenance, la socialisation étant plurielle et se développant tout au long de la vie.

6. Communication personnelle de Robert Chaudenson.

7. Les autres langues n'ont ni position officielle ni standard de référence.

8. Le français ivoirien présente effectivement des traits qui remettent en cause l'existence de zones totalement stables: ces faits sont hors français zéro, hors des lieux de variation bien connus du français selon mes recherches documentaires et de terrain. Plusieurs fois présentés lors de colloque, ils ont parfois semblé proches de faits connus dans d'autres espaces francophones ou créolophones, mais il s'avère qu'ils demeurent distincts de ceux-ci. Les exemples de cette section et de la suivante ont été revus pour ce texte avec des locuteurs experts du dioula tagbusi et du baoulé de Yamoussoukro (akué).

9. Cette différence n'est pas facilement perceptible. Cadiot (1991: 217) donne l'exemple suivant (il s'agit pour lui de contraster quant à et pour) : Les prix d'achat du mil et du sorgho ont bien été relevés. Quant à/pour celui du riz, il n'a pas été modifié. Dans ce cas, pour introduit un nouveau thème discursif et «ce que dit l'énoncé enchainé à un constituant pour $\mathrm{N}$ ne se construit pas directement comme un propos incident au denotatum de ce $N »$ (219). Ceci éloigne plus encore notre construction ivoirienne de pour $N$ de cette construction avec pour du français classique.

10. Sans reprendre ici toute la démonstration que j'ai menée ailleurs, je résume les principaux faits qui permettent de montrer des changements dans plusieurs langues à la fois. 
11. Le français tirailleur (début $20^{\mathrm{e}}$ siècle) est probablement issu lui-même du français parlé dans les comptoirs français du Sénégal aux $18^{\mathrm{e}}$ et $19^{\mathrm{e}}$ siècles, puis utilisé lors de la conquête de l'AOF à partir du Sénégal à la fin du $19^{\mathrm{e}}$ siècle.

12. Cette appellation a été utilisée lors du colloque "Autour du verbe », organisé par Ambroise Queffelec, Ingse Skattum et l'auteure de ce texte le 9 décembre 2009 à la MSH, Paris (Voir Boutin \& Kouamé 2011).

13. Je remercie ici les équipes PCF-CI, CFA-CI et CIEL-F-CI que j'ai eu la chance de diriger, composées de Aboa Alain Laurent, Beuseize André-Marie, Bli Bi Trazié Serge, Dodo Jean-Claude, Kouacou N'Goran Jacques, Kouadio Pierre Adou Kouakou, Kouamé Jean-Martial, Youant YvesMarcel.

14. Toutefois, un allongement du [i] final des infinitifs en -ir est susceptible de se faire entendre.

\section{RÉSUMÉS}

L'article se penche sur les influences entre les langues sur une aire plurilingue, dans le but de questionner la notion de convergence. Celle-ci présente a priori l'intérêt de tenir compte du fait que plusieurs facteurs sont présents ensemble et peuvent créer une situation propice au changement du fait de leur réunion. Par ailleurs, les situations plurilingues et pluriculturelles de l'Afrique d'aujourd'hui ont l'avantage d'être observables est vécues en direct et non à reconstituer à partir de recherches dans le passé. Après un examen général de la convergence des facteurs sociolinguistiques pour la Côte d'Ivoire, deux cas de réanalyses assez différents sont étudiés plus en détail dans le but d'explorer une possible convergence des langues : la possibilité pour des syntagmes prépositionnels d'avoir des fonctions de syntagmes nominaux, avec le cas particulier de pour, et la fonctionnalisation d'une forme verbale comme conditionnel périphrastique en français.

The article studies the influences between languages in a plurilingual area, in order to question the concept of convergence. This has the immediate advantage of taking into account the fact that several factors can together create a situation conducive to change by the very fact of their meeting. One of the goals of our contribution is to underline the impact of plurilingualism on the cognitive processing of languages by speakers. Indeed, postcolonial African territories make it possible to observe in real time what speakers do with languages, which is more advantageous than reconstituting situations from past research. The behaviors of multilingual speakers go beyond anything an observer of the language can imagine ex post. By multiplying the resources of their repertoire, plurilingualism multiplies the influx of possibilities of saying and ways of saying and, consequently, the factors of convergence so that a construction is privileged among others. However, there is immense heterogeneity and the practices have little stability (Boutin 2017), to the point that it is impossible to predict what, in the proliferation of syntactic and lexical creations, will remain as a result of code mixtures.

Numerous facts about the three languages most commonly in contact in Ivory Coast, French, Jula and Baule, show two levels of convergence: between several factors of sociolinguistic and communicational order, so that an innovation first appears in a single language (Kriegel 2018; Mufwene 2005), and then between languages, when a construction scheme carrying meaning in one or more of these languages has served as a model for reanalysis of shapes in one or more other languages. The notion of reanalysis that I use is close to that of re-functionalization and is 
easily conceived as a result of unguided acquisition processes. Reanalysis is the result of comprehension strategies, a reinterpretation of the lexicon and the role of morphemes or constructions (Cf. Kriegel 2003; Detges 2003) which leads to an innovation including a recategorization.

The first part examines the convergence of factors for the Ivory Coast while the following parts detail the study of two cases of rather different analysis, with the aim of exploring a possible convergence of languages. The more widely used languages, in this case Ivorian French, Jula and Baule have mutually changed due to several factors such as the wide knowledge of their main constructions, the way in which the speakers manage an approximate inter-understanding by formal tolerance and diversified reformulation, the popularity of Nouchi, a mixed slang, and the more or less consensual practices of alternating languages. It is by using their repertoire and their plurilingual competence that speakers have operated reanalyses or adaptations (Gumperz 1964, Gumperz \& Wilson 1971) by convergence between the various functionings of the languages present in the speeches.

The second part presents the possibility for prepositional phrases to have functions of noun phrases, such as dans la cour, bo kónon, àwlo nun (in the courtyard) in examples (1) to (3), in which speakers talk about plantains.

IF (1) c'est ici qui est mûr, dans la cour n'est pas mûr

PRES here REL COP ripe PREP DEF courtyard COP.NEG ripe

Jula (2) yàn lé mònnan, bo kónon man mòn

here COP ripe house in COP.NEG ripe

Baule (3) wàfan lo-li, àwlo nun wa-lo-man

here ripen-PFV courtyard interior AUX-ripe-NEG

(Those here are ripe, those in the courtyard are not ripe)

While in French, the adpositions, in this case prepositions (which often derive from relational nouns), form a completely distinct category from nouns, Baule, like other Akan languages, has no adpositions. Baule uses relational nouns likely to provide localization with their anteposed noun complement, when French, like Romance languages, uses locative prepositional phrases. Jula represents an intermediate case since it has formal postpositions which have no nominal property, and locative postpositions, of the same form and of the same construction as relational nouns, but no longer having the specifying properties of names. These three languages could detail the scale of grammaticalization of nouns in adpositions of Hopper \& Traugott (2003: 110-111) in a convergent process, without it being yet possible to say whether the change will be accelerated, for Baule for example, due to contact. On the other hand, speakers of Ivorian French who know the properties of languages such as Jula or Baule, easily grant nominal properties to locative prepositional phrases, even if the prepositions do not have a nominal origin as dans (1), seeming thus to downgrade the process.

The third part analyzes, beyond prepositional phrases which have functions of noun phrases, the particular case of pour, in French, of tá in Jula and of lic in Baule in examples like (4) to (6) in which the third person ( $35 \mathrm{SG}$ ) is a trader.

IF (4) je n'achète pas pour lui

1SG buy.IPFV.NEG PREP 3SG

Jula (5) $n$ téà tá sàn

1SG IPFV.NEG 3SG POSTP buy

Baule (6) $n$ to man $\boldsymbol{i}$ lié

1SG.NOM buy.IPFV NEG 3SG part

TIPA. Travaux interdisciplinaires sur la parole et le langage, 37 | 2021 
(I don't buy his merchandise)

The comparison with a vehicular French from the colonial period, of which we have written sources, and with Manding languages that have not known the vehicularization and contact that Jula has known, highlights various reanalysis processes making the properties of these morphemes converge.

The fourth part studies the functionalization of a verbal form into a periphrastic conditional in French, illustrated by examples (7) to (9) in West African French.

WAF (7) Si on partait en huitième de finale [...] on allait arriver

(If we started in the round of 16 [...] we would make it)

(8) Si j'étais riche sincèrement j'allais aider ceux qui sont dans le besoin

(If I was rich honestly I would help those in need)

(9) Donc vraiment si j'avais l'argent j'allais beaucoup aider ces enfants-là et leur maman

(So, if I really had the money I would help these children and their mom a lot).

In this case, no structural convergence is visible, but an influence of the languages in contact is possible at a deeper semantic level than the mere multilingualism of the speakers: the extralinguistic factors mentioned above converge to favor such reanalyses.

In conclusion, the notion of convergence does not always prove to be discriminating in the case of a multiplicity of factors likely to join at different levels to bring about linguistic change. It needs to be anchored in the much broader consideration of linguistic ecology, such as Mufwene (2008) and Ludwig, Pagel \& Mühlhäusler (2018) approach it. Their models try to show how these multiple factors interact, and are arguably better frameworks for going further in the analysis.

\section{INDEX}

Mots-clés : convergence de facteurs, plurilinguisme africain, changement linguistique, français de Côte d'Ivoire, dioula, baoulé

Keywords : convergence of factors, African plurilingualism, linguistic change, Ivory Coast French, Jula, Baule

\section{AUTEUR}

\section{AKISSI BÉATRICE BOUTIN}

Institut de Linguistique appliquée, UFHB, Abidjan \& Università Degli Studi di Roma "La Sapienza" boubeaki@gmail.com 